\title{
Construction of Mycobacterium tuberculosis cdd knockout and evaluation of invasion and growth in macrophages
}

\author{
Anne Drumond Villela ${ }^{1 /+}$, Valnês S Rodrigues-Junior ${ }^{1}$, Antônio Frederico Michel Pinto', \\ Virgínia Carla de Almeida Falcão, ${ }^{1,2}$, Zilpa Adriana Sánchez-Quitian ${ }^{1,2}$, Paula Eichler ${ }^{1}$, \\ Cristiano Valim Bizarro, ${ }^{1,2}$, Luiz Augusto Basso ${ }^{1,2,3}$, Diógenes Santiago Santos ${ }^{1,2}$
}

'Pontifícia Universidade Católica do Rio Grande do Sul, Centro de Pesquisas em Biologia Molecular e Funcional, Instituto Nacional de Ciência e Tecnologia em Tuberculose, Porto Alegre, RS, Brasil

${ }^{2}$ Pontifícia Universidade Católica do Rio Grande do Sul, Programa de Pós-Graduação em Biologia Celular e Molecular, Porto Alegre, RS, Brasil

${ }^{3}$ Pontifícia Universidade Católica do Rio Grande do Sul, Programa de Pós-Graduação em Medicina e Ciências da Saúde, Porto Alegre, RS, Brasil

Cytidine deaminase (MtCDA), encoded by $c d d$ gene (Rv3315c), is the only enzyme identified in nucleotide biosynthesis pathway of Mycobacterium tuberculosis that is able to recycle cytidine and deoxycytidine. An M. tuberculosis knockout strain for $c d d$ gene was obtained by allelic replacement. Evaluation of mRNA expression validated $c d d$ deletion and showed the absence of polar effect. MudPIT LC-MS/MS data indicated thymidine phosphorylase expression was decreased in knockout and complemented strains. The $c d d$ disruption does not affect M. tuberculosis growth both in Middlebrook 7H9 and in RAW 264.7 cells, which indicates that $c d d$ is not important for macrophage invasion and virulence.

Key words: Mycobacterium tuberculosis - cdd gene - cytidine deaminase

Tuberculosis is an infectious disease caused mainly by Mycobacterium tuberculosis. The World Health Organization estimated that 10.4 million people developed tuberculosis in 2015, resulting in 1.8 million deaths (WHO 2016). Although an efficient chemotherapy exists, affordable, short, effective and well-tolerated treatments for coadministration with anti-HIV agents, latent, drug-susceptible and drug-resistant tuberculosis are still needed to decrease the global incidence of the disease (Mdluli et al. 2015). The first step towards the search for novel therapeutic strategies is to better understand important metabolic pathways of the pathogen. Pyrimidine biosynthesis pathway provides pyrimidine nucleotides that are essential components of many biomolecules. Pyrimidine nucleotides in $M$. tuberculosis may be synthetised de novo from simple precursors, or may be obtained by the salvage pathway from preformed pyrimidine bases and nucleosides (Warner et al. 2014). While the de novo pathway is a high energy demanding process, the salvage pathway might be preferentially utilised under restricted energy availability (Villela et al. 2011).

Cytidine deaminase (MtCDA, EC 3.5.4.5) is encoded by $c d d$ gene (Rv3315c, Gene ID: 887975), and is within $c d d$-add operon with $d e o A$ (Rv3314c, thymidine phosphorylase, MtTP, EC 2.4.2.4) and add (Rv3313c, adenosine deaminase, MtAD, EC 3.5.4.4) genes (Roback et al. 2007). The $c d d$ gene was predicted to be non-essential by Himarl-based transposon mutagenesis in H37Rv (Sas-

doi: 10.1590/0074-02760170105

Financial support: INCT-TB (Brazil), Decit/SCTIE/MS-MCT-CNPq-FNDCT-

CAPES, CNPq (441720/2014-5 to ADV), BNDES (14.2.0914.1)

+ Corresponding author: anne.villela@pucrs.br

Received 15 March 2017

Accepted 30 May 2017 setti et al. 2003, Griffin et al. 2011). MtCDA is an important enzyme from the pyrimidine salvage pathway in $M$. tuberculosis that recycles cytidine and 2'-deoxycytidine for uridine and 2'-deoxyuridine synthesis, respectively (Sánchez-Quitian et al. 2010), and is the only enzyme identified by sequence homology in nucleotide biosynthesis pathway of $M$. tuberculosis that is able to rescue cytidine and deoxycytidine (Villela et al. 2011). Deoxyuridine can be converted to uracil by pyrimidine nucleoside phosphorylase (EC 2.4.2.4, deoA, Rv3314c) enzyme. Then, uracil phosphoribosyltransferase (EC 2.4.2.9, upp, Rv3309c) enzyme catalyses the conversion of uracil to uridine monophosphate (UMP), which is the precursor of all pyrimidine nucleotides (Villela et al. 2011). Uridine nucleosidase (EC 3.2.2.3) and uridine phosphorylase (EC 2.4.2.3) that convert uridine into uracil, and uridine kinase (EC 2.7.1.48) that catalyses the conversion of uridine into UMP, were not identified by sequence homology in M. tuberculosis genome (Cole et al. 1998).

Functional and structural studies of MtCDA enzyme were described previously (Sánchez-Quitian et al. 2010, 2015); however, no information about the direct essentiality of $c d d$ gene and its role in M. tuberculosis infection is available. Here, we describe the construction of an $M$. tuberculosis knockout strain for $c d d$ gene (KO), evaluation of mRNA expression of $c d d$, deoA and add genes, assessment of protein expression by MudPIT LC-MS/ MS, in vitro growth studies, and analysis of $c d d$ deletion in M. tuberculosis invasion and growth in a macrophage model of infection. To evaluate the effects of $c d d$ disruption on $M$. tuberculosis growth, the KO strain was compared with $M$. tuberculosis $\mathrm{H} 37 \mathrm{Rv}$ wild-type (WT) and complemented (CP) strains.

To construct the KO strain, a fragment of $1,788 \mathrm{bp}$ containing the $c d d$ gene (402 bp) with its flanking region (Fig. 1A) was amplified by polymerase chain reaction 
A

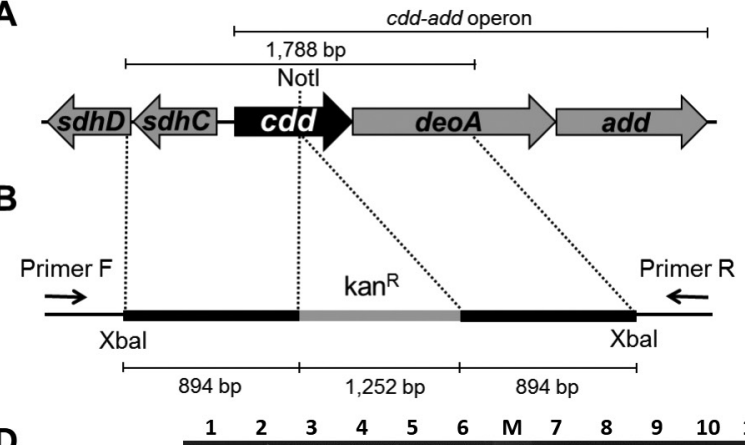

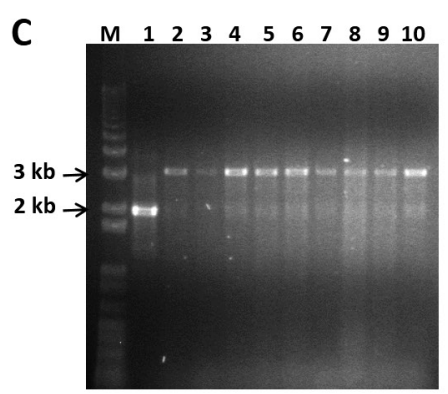

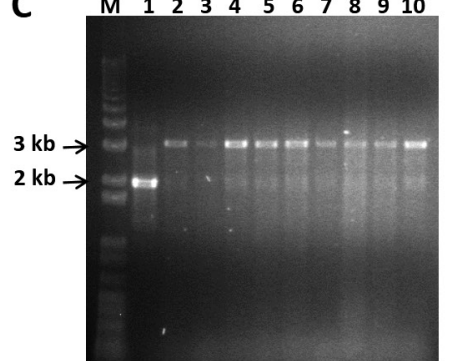

u

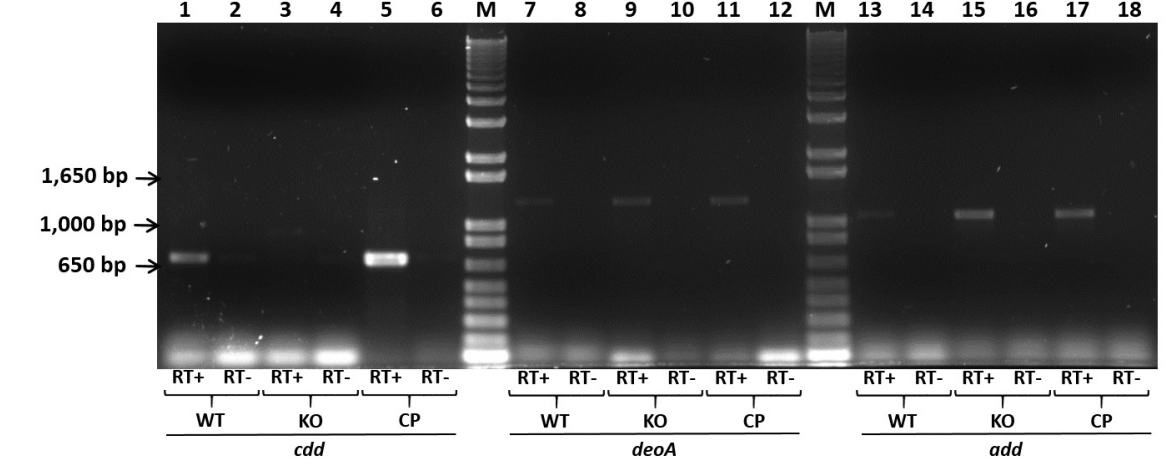

Fig. 1: genomic environment of $c d d$ gene in Mycobacterium tuberculosis (A), regions cloned into pPR27xylE vector (B), agarose gel electrophoresis of polymerase chain reaction (PCR) products from knockout clones (C), and mRNA expression of $c d d$, deoA, and add genes (D). (A) Genomic region of $c d d$ gene (402 bp) containing unique internal NotI site and flanking genes; the operon $c d d$-add is indicated. (B) The $c d d$ gene and flanking regions were amplified by PCR from $M$. tuberculosis H37Rv genomic DNA, and the $c d d$ gene was disrupted by the insertion of a kanamycin cassette $\left(\mathrm{kan}^{\mathrm{R}}\right)$ into $\mathrm{NotI}$ site $\left(c d d:: \mathrm{kan}^{\mathrm{R}}\right)$. The $c d d:: \mathrm{kan}^{\mathrm{R}}$ fragment was cloned into pPR27xylE vector using XbaI restriction site. Annealing regions of gene-specific screening primers forward (Primer F) and reverse (Primer R) for the possible knockout strains of $c d d$ gene are indicated. (C) Agarose gel electrophoresis of PCR products from possible knockout clones. M - molecular marker 1 kb plus DNA Ladder (Invitrogen), PCRs were carried out with: Lane 1 - M. tuberculosis H37Rv genomic DNA, Lanes 2-10 - possible knockout clones genomic DNA. (D) mRNA expression of $c d d$, deoA, and add genes. M - molecular marker $1 \mathrm{~kb}$ plus DNA Ladder (Invitrogen). Lanes 1-6: $c d d$ cDNA amplification (686 bp). Lanes 7-12: $d e o A$ cDNA amplification (1,284 bp). Lanes 13-18: add cDNA amplification (1,098 bp). cDNA synthesis from M. tuberculosis H37Rv (wild-type) was performed with reverse transcriptase enzyme (RT+) (lanes 1, 7, 13) or without (RT-) (lanes 2, 8, 14). cDNA synthesis from cdd knockout strain (KO) RT+ (lanes $3,9,15$ ) and RT- (lanes 4, 10, 16). cDNA synthesis from complemented strain (CP) RT+ (lanes 5, 11, 17) and RT- (lanes 6, 12, 18).

(PCR) from M. tuberculosis H37Rv genomic DNA, using primers forward (5'-tttttetagacccagegttgggcaacgaagt-3') and reverse (5'-tttttetagagcaccctcagccagcttcttg-3'), both containing $\mathrm{XbaI}$ restriction sites (in bold). The $1,788 \mathrm{bp}$ fragment was subsequently cloned into pUC19 using the $X b a I$ restriction site. The $c d d$ gene was disrupted by the insertion of a kanamycin cassette from pUC4K into unique internal enzyme restriction site NotI (Fig. 1B). Insert was released from pUC19 derivative vector by digestion with $X b a I$, and subcloned into XbaI linearized pPR27xylE vector (pPR27xylE::cdd kan) (Fig. 1B). The pPR27xylE plasmid contains a thermosensitive origin of replication, the $x y l E$ reporter gene, and the $s a c B$ counterselectable marker (Pelicic et al. 1997). M. tuberculosis H37Rv strain was transformed by electroporation with $\sim 2 \mu \mathrm{g}$ of pPR27xylE::cdd kan plasmid. Possible KO clones were selected in two steps, as described previously (Villela et al. 2017). Genomic DNA was isolated and PCRs were carried out using gene-specific screening primers forward (5'-gtgtctttgcggctgtagtc-3') and reverse (5'-gggcagttcatctccgtca-3') to determine whether the WT or the KO strain was present in the targeted chromosomal region (Fig. 1B). Among the nine clones screened for the KO of $c d d$ gene, all amplified a band of 3,293 bp compatible with a doublecrossover gene replacement event (Fig. 1C).
To obtain the CP strain, a fragment containing the $c d d$ gene, its upstream (183 bp) region containing the natural promoter, and $101 \mathrm{bp}$ downstream to $c d d$ was amplified by PCR from M. tuberculosis $\mathrm{H} 37 \mathrm{Rv}$ genomic DNA using primers forward (5'-ggggtctagattgtcgccgttgtattcacc-3') and reverse (5'-ggggtctagagtcggtataggcettgacga-3'), both containing XbaI restriction sites (in bold). Next, the amplicon was cloned into XbaI linearised pNIP40/b (pNIP40:: cdd), a mycobacteriophage Ms6-derived integrative vector (Freitas-Vieira et al. 1998), and the KO strain was transformed by electroporation with pNIP40:: cdd, as described previously (Villela et al. 2017). The stability of the mutation introduced by gene replacement in M. tuberculosis was evaluated by plating $\mathrm{KO}$ and $\mathrm{CP}$ strains on media with and without antibiotics. The difference between the colonies obtained on plates containing antibiotics was not significant when compared with the ones obtained on plates without antibiotic, which indicates that the introduced mutation is stable (data not shown).

As mentioned previously, the $c d d$ together with $d e o A$ and $a d d$ genes are predicted to form an operon. Thus, to investigate the effect of $c d d$ disruption on $d e o A$ and add genes, levels of mRNA expression of $c d d, d e o A$, and add genes were monitored in WT, KO and CP strains. Ten milliliters of M. tuberculosis cultures were grown up to 
an $\mathrm{OD}_{600}$ of $0.6-0.8$. The cell pellet was suspended in 1 $\mathrm{mL}$ of TRIzol (Invitrogen, Carlsbad, CA, USA), and disrupted in $2 \mathrm{~mL}$ screw-cap tubes containing $0.1 \mathrm{~mm}$ silica spheres using a L-Beader 3 (Loccus Biotecnologia, Cotia, Brazil). The aqueous phase was extracted with $200 \mu \mathrm{L}$ of chloroform, and RNA was precipitated with $500 \mu \mathrm{L}$ of isopropanol. Remaining DNA in RNA samples was digested with DNAse (RNAse free DNAse set, QIAgen, Hilden, Germany) and RNA samples were purified by using an RNA purification kit (RNeasy mini kit, QIAgen, Hilden, Germany). Synthesis of the first strand of cDNA was performed using $0.5 \mu \mathrm{g}$ RNA from $M$. tuberculosis $\mathrm{H} 37 \mathrm{Rv}, \mathrm{KO}$, and CP strains as template and random hexamers as primers, following the instructions in the SuperScript III First-Strand Kit protocol (Invitrogen, Carlsbad, CA, USA). An aliquot of cDNA synthesis reaction was used to amplify $c d d$ gene with primers $c d d \mathrm{~F}$ (5'-ggggtctagattgtcgcegttgtattcacc-3') and $c d d \mathrm{R}$ (5'-ggggtctagagtcggtataggecttgacga-3'), deo $A$ gene with primers deo $A$ F (5'- cgcatatgaccgacttcgcattcgacgeccc- $\left.3^{\prime}\right)$ and $d e o A \mathrm{R}$ (5'-agaagctttcagacgatccgatcgacgattagc-3'), and add gene with primers $a d d \mathrm{~F}$ (5'-gtcatatgaccgctgcgecgaccetgcag-3') and add R (5'- ctggatcctcactcgetgtgacccatgagc -3'). Amplification products were analysed in $1 \%$ agarose gels. As shown in Fig. 1D, no $c d d$ expression was observed in KO strain (lane 3), but was detected in WT (lane 1) and $\mathrm{CP}$ (lane 5) strains. The $d e o A$ and $a d d$ mRNA expression were observed in all strains (Fig. 1D), which indicated the disruption of $c d d$ by insertion of a kanamycin cassette did not exert a polar effect on the expression of these genes. No or minor DNA contamination was observed on negative controls, in which the cDNA synthesis was performed without the reverse transcriptase enzyme (Fig. 1D).

Although the transcription of deoA and add genes were not affected by $c d d$ deletion in KO strain, the expression of downstream genes to $c d d$ might be affected at the protein level if the translation is coupled. Coupled translation was observed in Escherichia coli, where the translation efficiency of one gene affects indirectly the translational level of downstream genes within an operon, potentially causing a strong phenotype (Levin-Karp et al. 2013). The fact that the start and stop codons of $c d d$, $d e o A$ and add genes overlap strengthens the possibility of a coupled expression. Therefore, Liquid Chromatography and Tandem Mass Spectrometry (LC-MS/ MS) and Multidimentional Protein Identification Technology (MudPIT) analyses were performed to evaluate MtCDA, MtTP, and MtAD expression levels in WT, KO and CP strains. Cytoplasmic fractions of mycobacterial protein extracts from WT, KO and CP strains were obtained by ultracentrifugation, as described (Gunawardena et al. 2013). Chloroform/methanol protein precipitation was performed in WT, KO and CP cytoplasmic fraction $(200 \mu \mathrm{g})$ according to (Wessel \& Flügge 1984). Protein pellets were resuspended in $100 \mathrm{mM}$ Tris $\mathrm{HCl}$ $\mathrm{pH} 8.5$ containing $8 \mathrm{M}$ urea, and digested according to Klammer and MacCoss (2006). Chromatographic separations were performed on a nanoLC Ultra 1D plus with autosampler (Sciex, Framingham, MA, USA) connected to a LTQ-XL Orbitrap Discovery hybrid instrument (Thermo Fisher Scientific, Waltham, MA, USA) through a nanoeletrospray ion source (Thermo Fisher

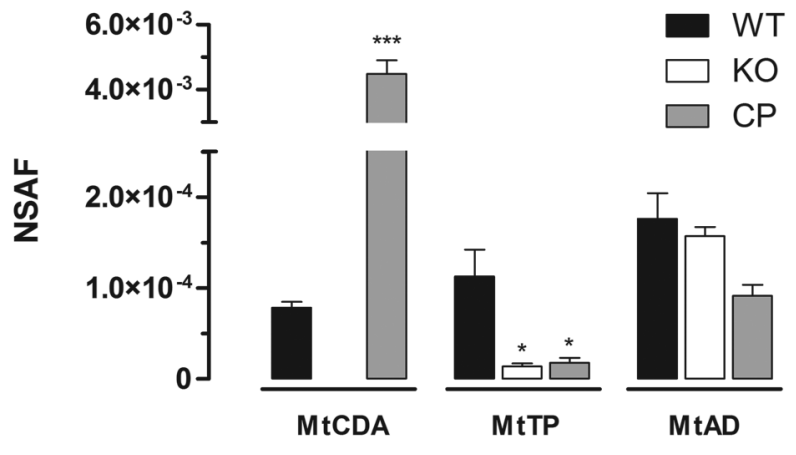

Fig. 2: evaluation of MtCDA, MtTP and MtAD expression. MtCDA, MtTP and MtAD normalised spectral abundance factor (NSAF) in wild-type (WT), knockout strain (KO) and complemented strain (CP) cytoplasmic fractions identified by Multidimentional Protein Identification Technology (MudPIT). Bars represent average identification in technical triplicates. Asterisks represent significant differences between WT and KO or CP strains of each protein by one-way ANOVA analysis followed by Bonferroni post-test, ${ }^{*} \mathrm{p}<0.05, * * * \mathrm{p}<0.001$.

Scientific, Waltham, MA, USA). Biphasic MudPIT columns and capillary analytical columns were prepared in house (Villela et al. 2015). MudPIT analysis was carried out according to Wolters (Wolters et al. 2001). Analyses were performed in technical triplicates. Data was collected with one MS1 full-scan in the Orbitrap (400-1600 $\mathrm{m} / \mathrm{z}$ range; 30,000 resolution) followed by data dependent CID MS/MS spectra of the eight most intense ions in the ion trap, with dynamic exclusion applied. Mass spectra were searched for candidate peptides with the software Comet (Eng et al. 2012) in the platform PatternLab for Proteomics (Carvalho et al. 2016). The database contained a non-redundant $M$. tuberculosis reference proteome (ID UP000001584, www.uniprot.org) and the reverse sequences of all entries. The validity of the peptide spectra matches (PSMs) was assessed using the module Search Engine Processor (SEPro) from Patternlab for Proteomics, with a false discovery rate of $1 \%$. Normalised spectral abundance factor (NSAF) was calculated according to Zybailov et al. (2006), and data were evaluated with the one-way ANOVA analysis, followed by Bonferroni's post-test, using GraphPad Prism 5.0. Differences were considered significant at the $95 \%$ level of confidence. With average outputs of 76,880 spectra and 12,700 unique peptides per MudPIT run, the proteomic pipeline applied here identified a total of 2075 mycobacterial proteins in the cytoplasmic fraction of the three strains, considering only proteins identified consistently in three technical replicates (data not shown). Spectra matching peptides from MtAD and MtTP were identified in $\mathrm{WT}, \mathrm{KO}$ and $\mathrm{CP}$ protein extracts. A significant decrease in the level of MtTP (encoded by deoA gene) was observed in both $\mathrm{KO}$ and $\mathrm{CP}$ strains when comparing with WT strain (Fig. 2). This result suggests the occurrence of translational coupling between the neighboring genes $c d d$ and $d e o A$. On the other hand, the levels of MtAD, encoded by the third gene in the operon (add) (Fig. 1A), was not affected by the disruption of $c d d$ gene (Fig. 2). This difference in translation interdependency might be explained by the fact that the disrupted $c d d$ gene is closer to $d e o A$ than to $a d d$ (Fig. 1A). MtCDA 
peptides were identified in both WT and CP but not in KO protein extracts (Fig. 2), indicating that the disruption of $c d d$ gene abolishes the production of MtCDA protein in the KO strain. MudPIT LC-MS/MS results are in agreement with mRNA expression evaluation, in which $c d d$ gene expression was identified in WT and CP strains but was absent in KO strain (Fig. 1D). MtCDA peptides were only detected in WT strain using ultracentrifugation for protein fractionation and MudPIT for peptide fractionation and identification. Seven spectral counts of two MtCDA unique peptides were identified in the WT strain technical triplicates. In the CP strain technical triplicates, 464 spectral counts of $10 \mathrm{MtCDA}$ unique peptides were identified, a 66-fold difference in the levels of MtCDA in WT and CP strains. The difference in MtCDA levels between WT and CP strains could be explained by the fact that, though expressed by MtCDA natural promoter, the complemented copy of $c d d$ gene is integrated in a different genome region.

The growth rate of the $\mathrm{WT}, \mathrm{KO}$, and $\mathrm{CP}$ strains were compared to determine whether $c d d$ disruption leads to alterations during in vitro cultivation of $M$. tuberculosis. Growth curves were started at an optical density at $600 \mathrm{~nm}$ $\left(\mathrm{OD}_{600}\right)$ of 0.01 in Middlebrook 7H9 10\% OADC 0.05\% tween-80 containing proper antibiotics, in triplicate, at $37^{\circ} \mathrm{C}, 80 \mathrm{rpm}$. Aliquots were removed from each culture at different time points and the $\mathrm{OD}_{600}$ was determined. As shown in Fig. 3A, the three strains have a similar pattern of growth when grown in Middlebrook 7H9 medium.

To examine whether the $c d d$ gene was important for invasion and growth in phagocytic cells, we determined the bacterial loads of the WT, KO, and CP strains by using the macrophage model of infection. RAW 264.7 macrophage cell line was cultured and infected with WT, KO or CP M. tuberculosis strains as described previously (Villela et al. 2017), with minor modifications. Briefly, infection of macrophages was performed at a multiplicity of infection of $1: 1$ (bacteria/macrophage) at $37^{\circ} \mathrm{C}$ with $5 \% \mathrm{CO}_{2}$. After $18 \mathrm{~h}$, infection was terminated, and at this time point, 3, 7, 14 and 21 days of incubation, infected macrophages were lysed (Rodrigues-Junior et al. 2014), and plated on Middlebrook 7H10 agar supplemented with
$10 \%$ OADC. Colony-forming unit (CFU) was evaluated after incubation of plates for three weeks at $37^{\circ} \mathrm{C}$. This experiment was performed in triplicate, and the results were expressed as mean numbers of the logarithms of CFU per well, and were evaluated with the two-way ANOVA analysis, followed by Bonferroni's post-test, using GraphPad Prism 5.0. Differences were considered significant at the $95 \%$ level of confidence. As shown in Fig. 3B, no significant differences were observed in intracellular growth among WT, KO, and CP strains in macrophages after $18 \mathrm{~h}$, three, seven and 21 days after infection. A decrease in bacterial load of KO strain was observed after 14 days of macrophage infection; however, compared to WT, it was not statistically significant (Fig. 3B). Although the decrease in bacterial load of KO strain after 14 days of macrophage infection was shown to be statistically significant $(\mathrm{p}<0.05)$ when compared with CP strain, the analysis of the area under the curve revealed very similar intracellular bacterial growth for WT (total area mean \pm standard error of the mean: 23.25 $\pm 0.47), \mathrm{KO}(23.32 \pm 0.27)$, or CP $(23.06 \pm 0.11)$ strains. Therefore, these data suggest that the disruption of $c d d$ gene does not affect the M. tuberculosis growth in RAW 264.7 cells in the experimental conditions employed here.

In conclusion, a $M$. tuberculosis $\mathrm{KO}$ strain for $c d d$ gene was constructed by allelic replacement. The $\mathrm{CP}$ strain was obtained by transforming the $\mathrm{KO}$ with pNIP40:: cdd plasmid that expresses $c d d$ gene from its natural promoter. The $c d d$ deletion was validated at the RNA level, and was confirmed at the protein level by MudPIT LC-MS/MS. The disruption of $c d d$ gene did not affect the mRNA expression of $d e o A$ and $a d d$ genes, both located downstream of $c d d$ on the same operon. However, MudPIT LC-MS/MS data indicated the MtTP protein level was decreased in both $\mathrm{KO}$ and $\mathrm{CP}$ strains, when compared with its level in WT strain, which could be explained by a translational coupling between $c d d$ and $d e o A$ genes. The $M$. tuberculosis growth kinetics in Middlebrook 7H9 medium was not affected by the disruption of $c d d$ gene. The results for RAW 264.7 cells suggest that the $c d d$ gene product plays no role in $M$. tuberculosis invasion, growth and virulence in macro-
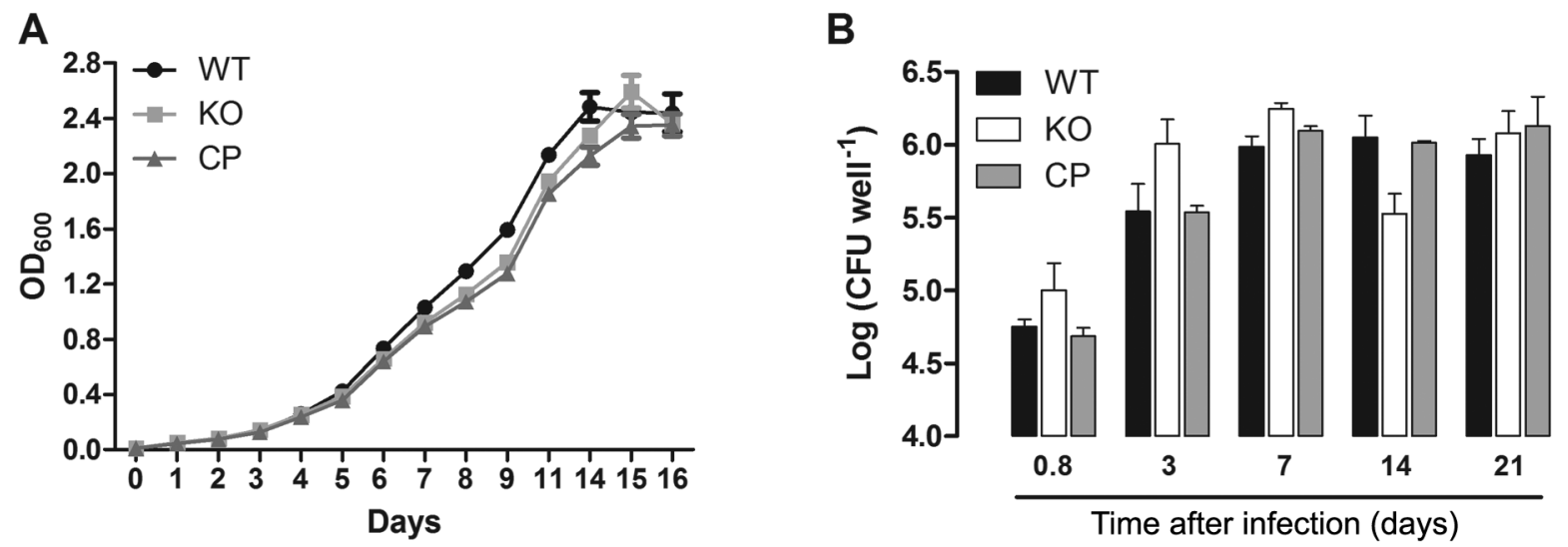

Fig. 3: growth curve (A) and macrophage infection (B) with wild-type H37Rv (WT), knockout for $c d d$ gene (KO), and complemented (CP) Mycobacterium tuberculosis strains. (A) Growth curves were started at an $\mathrm{OD}_{600}$ of 0.01 in Middlebrook $7 \mathrm{H} 9$ medium, in triplicate. (B) The macrophage infection results are expressed as mean numbers of the logarithms of colony-forming unit (CFU) per well of each strain of three independent measurements. 
phages. Even though the recycling of nucleotides and nucleosides might represent a significant energy saving for the cell, the MtCDA activity is not required in the context of in vitro growth and macrophage invasion and infection by M. tuberculosis under the experimental conditions employed here. These findings could be explained by the redundancy found in nucleotide metabolism of M. tuberculosis. Although MtCDA is the only enzyme identified by sequence homology in $M$. tuberculosis nucleotide biosynthesis pathway that is able to rescue cytidine and deoxycytidine (Villela et al. 2011), there are enzymes from de novo pathway that synthesize all pyrimidine nucleotides from simple precursors (Warner et al. 2014). These enzymes might compensate for the absence of $c d d$ gene in $\mathrm{KO}$ strain. However, it should be pointed out that studies under hypoxic and/or nutrient limitation conditions that likely mimic the environment in which latent bacilli survive should be pursued to provide a solid experimental basis for the role of $c d d$ gene product. Different results of gene essentiality and importance for M. tuberculosis growth and survival might be obtained under different experimental conditions.

\section{ACKNOWLEDGEMENTS}

To Dr Mary Jackson and Dr Brigitte Gicquel for providing the pNIP40/b and pPR27xylE plasmids.

\section{AUTHORS' CONTRIBUTION}

ADV constructed and validated the Mycobacterium tuberculosis knockout and complemented strains, performed the growth curve, and wrote the manuscript; ADV and VSRJ performed macrophage infection experiments; AFMP, PE and CVB carried out MudPIT LC-MS/MS experiments and performed data analysis; VCAF participated in all biosafety level 3 experiments; ZASQ constructed the plasmids; LAB contributed in the analysis of results and manuscript revision; and DSS conceived the study and participated in its design and coordination.

\section{REFERENCES}

Carvalho PC, Lima DB, Leprevost FV, Santos MD, Fischer JS, Aquino PF, et al. Integrated analysis of shotgun proteomic data with PatternLab for proteomics 4.0. Nat Protoc. 2016; 11(1): 102-17.

Cole ST, Brosch R, Parkhill J, Garnier T, Churcher C, Harris D, et al. Deciphering the biology of Mycobacterium tuberculosis from the complete genome sequence. Nature. 1998; 393(6685): 537-44.

Eng JK, Jahan TA, Hoopmann MR. Comet: an open source tandem mass spectrometry sequence database search tool. Proteomics. 2012; 13(1): 22-4.

Freitas-Vieira A, Anes E, Moniz-Pereira J. The site-specific recombination locus of mycobacteriophage Ms6 determines DNA integration at the tRNA(Ala) gene of Mycobacterium spp. Microbiology. 1998; 144(12): 3397-406.

Griffin JE, Gawronski JD, Dejesus MA, Ioerger TR, Akerley BJ, Sassetti CM. High-resolution phenotypic profiling defines genes essential for mycobacterial growth and cholesterol catabolism. PLoS Pathog. 2011; 7(9): e1002251.

Gunawardena HP, Feltcher ME, Wrobel JA, Gu S, Braunstein M, Chen X. Comparison of the membrane proteome of virulent $M y$ cobacterium tuberculosis and the attenuated Mycobacterium bovis BCG vaccine strain by label-free quantitative proteomics. J Proteome Res. 2013; 12(12): 5463-74.
Klammer AA, MacCoss MJ. Effects of modified digestion schemes on the identification of proteins from complex mixtures. J Proteome Res. 2006; 5(3): 695-700.

Levin-Karp A, Barenholz U, Bareia T, Dayagi M, Zelcbuch L, Antonovsky N, et al. Quantifying translational coupling in E. coli synthetic operons using RBS modulation and fluorescent reporters. ACS Synth Biol. 2013; 2(6): 327-36.

Mdluli K, Kaneko T, Upton A. The tuberculosis drug discovery and development pipeline and emerging drug targets. Cold Spring Harb Perspect Med. 2015; 5(6): a021154.

Pelicic V, Jackson M, Reyrat JM, Jacobs Jr WR, Gicquel B, Guilhot C. Efficient allelic exchange and transposon mutagenesis in Mycobacterium tuberculosis. Proc Natl Acad Sci USA. 1997; 94(20): 10955-60.

Roback P, Beard J, Baumann D, Gille C, Henry K, Krohn S, et al. A predicted operon map for Mycobacterium tuberculosis. Nucleic Acids Res. 2007; 35(15): 5085-95.

Rodrigues-Junior V, dos Santos A, Villela AD, Belardinelli JM, Morbidoni HR, Basso LA, et al. IQG-607 abrogates the synthesis of mycolic acids and displays intracellular activity against $\mathrm{Myco-}$ bacterium tuberculosis in infected macrophages. Int J Antimicrob Agents. 2014; 43(1): 82-5.

Sánchez-Quitian ZA, Rodrigues-Junior V, Rehm JG, Eichler P, Trivella DBB, Bizarro CV, et al. Functional and structural evidence for the catalytic role played by glutamate- 47 residue in the mode of action of Mycobacterium tuberculosis cytidine deaminase. RSC Adv. 2015; 5: 830-40.

Sánchez-Quitian ZA, Schneider CZ, Ducati RG, de Azevedo Jr WF, Bloch Jr C, Basso LA, et al. Structural and functional analyses of Mycobacterium tuberculosis Rv3315c-encoded metal-dependent homotetrameric cytidine deaminase. J Struct Biol. 2010; 169(3): 413-23.

Sassetti CM, Boyd DH, Rubin EJ. Genes required for mycobacterial growth defined by high density mutagenesis. Mol Microbiol. 2003; 48(1): 77-84.

Villela AD, Eichler P, Pinto AFM, Rodrigues-Junior V, Yates III JR, Bizarro CV, et al. Gene replacement and quantitative mass spectrometry approaches validate guanosine monophosphate synthetase as essential for Mycobacterium tuberculosis growth. Biochem Biophys Rep. 2015; 4: 277-82.

Villela AD, Rodrigues-Junior VS, Pinto AFM, Wink PL, SánchezQuitian ZA, Petersen GO, et al. Characterisation of iunH gene knockout strain from Mycobacterium tuberculosis. Mem Inst Oswaldo Cruz. 2017; 112(3): 203-8.

Villela AD, Sánchez-Quitian ZA, Ducati RG, Santos DS, Basso LA. Pyrimidine salvage pathway in Mycobacterium tuberculosis. Curr Med Chem. 2011; 18(9): 1286-98.

Warner DF, Evans JC, Mizrahi V. Nucleotide metabolism and DNA replication. Microbiol Spectrum. 2014; 2(5): doi: 10.1128/microbiolspec.MGM2-0001-2013.

Wessel D, Flügge UI. A method for quantitative recovery of protein in dilute solution in the presence of detergents and lipids. Anal Biochem. 1984; 138(1): 141-3.

WHO - World Health Organization. Global tuberculosis report 2016. Geneva: WHO; 2016.

Wolters DA, Washburn MP, Yates 3rd JR. An automated multidimensional protein identification technology for shotgun proteomics. Anal Chem. 2001; 73(23): 5683-90.

Zybailov B, Mosley AL, Sardiu ME, Coleman MK, Florens L, Washburn MP. Statistical analysis of membrane proteome expression changes in Saccharomyces cerevisiae. J Proteome Res. 2006; 5(9): 2339-47. 